\title{
A Topological Approach to Bend-Twist Maps with Applications
}

\author{
Anna Pascoletti and Fabio Zanolin \\ Department of Mathematics and Computer Science, University of Udine, via delle Scienze 206, \\ 33100 Udine, Italy \\ Correspondence should be addressed to Fabio Zanolin, fabio.zanolin@dimi.uniud.it \\ Received 26 May 2011; Accepted 21 July 2011 \\ Academic Editor: Leonid Berezansky
}

Copyright (C) 2011 A. Pascoletti and F. Zanolin. This is an open access article distributed under the Creative Commons Attribution License, which permits unrestricted use, distribution, and reproduction in any medium, provided the original work is properly cited.

In this paper we reconsider, in a purely topological framework, the concept of bend-twist map previously studied in the analytic setting by Tongren Ding in (2007). We obtain some results about the existence and multiplicity of fixed points which are related to the classical PoincaréBirkhoff twist theorem for area-preserving maps of the annulus; however, in our approach, like in Ding (2007), we do not require measure-preserving conditions. This makes our theorems in principle applicable to nonconservative planar systems. Some of our results are also stable for small perturbations. Possible applications of the fixed point theorems for topological bend-twist maps are outlined in the last section.

\section{Introduction, Basic Setting and Preliminary Results}

The investigation of twist maps defined on annular domains can be considered as a relevant topic in the study of dynamical systems in two-dimensional manifolds. Twist maps naturally appear in a broad number of situations, and thus they have been widely considered both from the theoretical point of view and for their significance in various applications which range from celestial mechanics to fluid dynamics.

One of the most classical examples of a fixed point theorem concerning twist maps on an annulus is the celebrated Poincaré-Birkhoff "twist theorem," also known as the "Poincaré last geometric theorem." It asserts the existence of at least two fixed points for an areapreserving homeomorphism $\phi$ of a closed planar annulus

$$
A[a, b]:=\left\{(x, y) \in \mathbb{R}^{2}: a \leq\|(x, y)\| \leq b\right\}
$$

$(0<a<b)$ onto itself which leaves the inner boundary $A_{i}:=\{z \in A[a, b]:\|z\|=a\}$ and the outer boundary $A_{o}:=\{z \in A[a, b]:\|z\|=b\}$ invariant and rotates $A_{i}$ and $A_{o}$ in 
the opposite sense (this is the so-called twist condition at the boundary). The Poincare-Birkhoff fixed point theorem was stated (and proved in some special cases) by Poincaré [1] in 1912, the year of his death. In 1913 [2], Birkhoff, with an ingenious application of the index of a vector field along a curve, gave a proof of the existence of a fixed point (see also [3]). A complete description of Birkhoff's approach, with also the explanation how to obtain a second fixed point, can be found in the expository article by Brown and Neumann [4]. The history of the "twist" theorem and its generalizations and developments is quite interesting but impossible to summarize in few lines. After about hundred years of studies on this topic, some controversial "proofs" of its extensions have been settled only recently. We refer the interested reader to [5] where the part of the story concerning the efforts of avoiding the condition of boundary invariance is described. In this connection, we also recommend the recent works by Martins and Ureña [6] and by Le Calvez and Wang [7] as well as the references therein.

Our study for the present paper is motivated by a recent approach considered by Ding in $[8$, Chapter 7] for the proof of the Poincare-Birkhoff theorem for analytic functions. In the same chapter, a concept of bend-twist map is introduced. Roughly speaking, analytic bendtwist maps are those analytic twist maps in which the radial displacement $\|\phi(z)\|-\|z\|$ changes its sign on a Jordan closed curve which is noncontractible in the annulus and where the angular displacement vanishes. Our goal is to extend Ding's definition to a pure topological setting and obtain some fixed point theorems for continuous bend-twist maps. Our results do not require any regularity on the maps involved. Moreover, we do not assume hypotheses like homeomorphism, area preserving, or invariance of the boundaries, and, as an additional feature, some of our results are stable under small perturbations. These facts, in principle, suggest the possibility to produce some new applications to planar differential systems which are not conservative. Our main existence theorem (see Theorem 2.7) follows from the Borsuk separation theorem and Alexander's lemma which we have extensively applied in a recent paper [9]. Our result partially extends Ding's theorem to the nonanalytic setting. The main difference between Theorem 2.7 and the corresponding theorem in [8] lies on the fact that we obtain at least one fixed point, whence two fixed points are given in [8]. On the other hand, we show, by a simple example, that only one fixed point may occur in some situations. Both in our case and in Ding's, the main hypothesis for the bend-twist theorem is a rather abstract one. Hence some more applicable corollaries, in the line of [8], are provided (see Theorem 2.9 and Corollary 2.10). In a final section we outline an application of our results to the periodic problem for some nonlinear ordinary differential equations.

We end this introduction with some definition and basic results which will be useful in the subsequent sections.

Definition 1.1. A topological space $X$ is called a topological annulus if it is homeomorphic to a planar annulus $A[a, b]$ with $A[a, b]$ defined as in (1.1).

Let $X$ be a topological annulus, and let $\eta: A[a, b] \rightarrow \eta(A[a, b])=X$ be a homeomorphism. As a consequence of Schoenflies's theorem [10], the set $\eta(\partial A[a, b])$ is independent of the choice of the homeomorphism $\eta$. We call the set $\eta(\partial A[a, b])$ the contour of $X$ and denote it by $\vartheta X$. Clearly, for a topological annulus $X$ embedded in $\mathbb{R}^{2}$, the contour of $X$ coincides with the boundary of $X$. The contour of $X$ consists of two connected components which are closed arcs (Jordan curves) since they are homeomorphic to $S^{1}$. We call such closed $\operatorname{arcs} X_{i}$ and $X_{o}$. For a planarly embedded topological annulus, they could be chosen as the inner and the outer boundaries of the annulus. In such a special case, the bounded 
component of $\mathbb{R}^{2} \backslash X$ turns out to be an open simply connected set $D=D(X)$ with $\partial D=X_{i}$ and cl $D=D \cup X_{i}$ homeomorphic to the closed unit disc (this can be proved by means of the Jordan-Schoenflies theorem [10]). On the other hand, in the general setting, speaking of inner and outer boundaries is meaningless; yet we keep this terminology. Finally, we define the interior of $X$ as

$$
\operatorname{int} X:=X \backslash \vartheta X \text {. }
$$

Slightly modifying an analogous definition of Berarducci et al. [11, Definition 2.1] we give the following.

Definition 1.2. Let $X$ be a topological space and let $A, B \subset X$ be two nonempty disjoint sets. Let also $S \subset X$. We say that $S$ cuts the paths between $A$ and $B$ if $S \cap \bar{\gamma} \neq \emptyset$, for every continuous map (from now on, a path) $\gamma:[0,1] \rightarrow X$ such that $\gamma(0) \in A$ and $\gamma(1) \in B$. The notation $\bar{\gamma}:=\gamma([0,1])$ is used.

In order to simplify the presentation, we write $S: A \nmid B$ to express the fact that $S$ cuts the paths between $A$ and $B$. To make Definition 1.2 meaningful, we implicitly assume that there exists at least a path $\gamma$ in $X$ connecting $A$ with $B$ (otherwise, we could take $S=\emptyset$, or $S$ any subset of $X$ ). Such assumption will always be satisfied in the sequel. Clearly, if a set $S$ satisfies the cutting property of Definition 1.2, then also its closure cl $S$ cuts the paths between $A$ and $B$. Therefore, we usually assume $S$ closed.

Let $X$ be an arcwise connected topological space. We say that a set $C \subset X$ is essentially embedded in $X$ if the inclusion

$$
i_{C}: C \longrightarrow X, \quad i_{C}(x)=x, \forall x \in C
$$

is not homotopic to a constant map in $X$.

The next result is a corollary of the Borsuk separation theorem [12, Theorem 6-47] adapted to our context. For a detailed proof, see also [9].

Lemma 1.3. Let $X$ be a topological annulus, and let $S \subset X$ be a closed set. Then $S$ is essentially embedded in $X$ if and only if $S: X_{i} \nmid X_{o}$.

For our applications to bend-twist maps we also need a more refined version of the above result which reads as follows.

Lemma 1.4. Let $X$ be a topological annulus and let $S \subset X$ be a closed set such that $S: X_{i} \nmid X_{o}$. Then there exists a compact, connected set $C \subset S$ such that $C: X_{i} \nmid X_{o}$ (and, therefore, $C$ is essentially embedded in X).

Proof. First of all we claim that there exists a closed set $C \subset S$ such that $C: X_{i} \nmid X_{o}$, with $C$ minimal with respect to the cutting property. This follows from a standard application of Zorn's lemma (see [9] for the details). Suppose, by contradiction, that $C$ is not connected, and let $C_{1}, C_{2} \subset C$ be two closed nonempty disjoint sets with $C_{1} \cup C_{2}=C$. Since $C$ is minimal and $C_{1}, C_{2}$ are proper subsets of $C$, there exist two paths $\gamma_{1}, \gamma_{2}$ in $X$ which connects $X_{i}$ to $X_{o}$ and such that $\gamma_{j}$ avoids $C_{j}$ (for $j=1,2$ ). Then, by Alexander's lemma [13] there exists a path $\gamma:[0,1] \rightarrow X$ with $\gamma(0) \in X_{i}$ and $\gamma(1) \in X_{o}$ with $\bar{\gamma} \cap C=\emptyset$, contradicting the assumption that $C: X_{i} \nmid X_{o}$. The continuum $C$ is also essentially embedded in $X$ by Lemma 1.3. 
In the sequel we denote by $\Pi$ the standard covering projection of $\mathbb{R} \times \mathbb{R}_{0}^{+}$onto $\mathbb{R}^{2} \backslash\{0\}$, defined by the polar coordinates $\Pi(\theta, \rho)=(\rho \cos \theta, \rho \sin \theta)$.

\section{Bend-Twist Maps}

In this section, we reconsider, in a purely topological framework, the concept of bend-twist map introduced by Ding in [8]. The basic setting in [8] considers a pair of starlike planar annuli $A, A^{*}$ with $A \subset A^{*}$ and a continuous map $f: A \rightarrow A^{*}$. Without loss of generality (via a translation of the origin), one can always assume that $0=(0,0)$ belongs to the open set $D\left(A^{*}\right)$. Accordingly, our basic setting will be the following.

Let $X \subset \mathbb{R}^{2}$ be a topological annulus (embedded in the plane) with $0 \in D(X)$. Passing to the covering space $(\theta, \rho)$, we have that the inner and outer boundaries of $X$ are lifted to the lines $J_{i}:=\Pi^{-1}\left(X_{i}\right)$ and $J_{o}:=\Pi^{-1}\left(X_{o}\right)$ which are periodic in the sense that $(\theta, \rho) \in J$ if and only if $(\theta+2 \pi, \rho) \in J$, for $J=J_{i}, J_{o}$. In [8] the boundaries are assumed to be starlike, that is, both $J_{i}$ and $J_{o}$ are graphs of $2 \pi$-periodic functions $\lambda_{i}, \lambda_{o}: \mathbb{R} \rightarrow \mathbb{R}_{0}^{+}, \theta \mapsto \rho=\lambda(\theta)\left(\right.$ for $\lambda=\lambda_{i}, \lambda_{o}$ ) with $\lambda_{i}(\theta)<\lambda_{o}(\theta)$, for all $\theta \in \mathbb{R}$. The condition about the strictly star-shaped boundaries of $X$ is crucial for entering in the setting of the Poincare-Birkhoff theorem (see $[6-8,14]$ ). However, we will not assume it unless when explicitly required.

Let $\phi=\left(\phi_{1}, \phi_{2}\right): X \rightarrow \mathbb{R}^{2} \backslash\{0\}$ be a continuous map. By the theory of covering projections [15] there exists a (continuous) lifting $\tilde{\phi}$ of $\phi$ defined on $\Pi^{-1}(X) \rightarrow \mathbb{R} \times \mathbb{R}_{0}^{+}$such that

$$
\phi \circ \Pi=\Pi \circ \tilde{\phi}
$$

By definition, given a lifting $\tilde{\phi}$ of $\phi$, all the other liftings of $\phi$ are of the form

$$
(\theta, \rho) \longmapsto \tilde{\phi}(\theta, \rho)+(2 k \pi, 0),
$$

for some $k \in \mathbb{Z}$. We assume that $\tilde{\phi}$ can be expressed as

$$
\tilde{\phi}:(\theta, \rho) \longmapsto(\theta+\Theta(\theta, \rho), R(\theta, \rho)),
$$

where $\Theta, R$ are continuous real-valued functions defined on $\Pi^{-1}(X)$ and $2 \pi$-periodic in the $\theta$-variable. We also introduce an auxiliary function $\Upsilon$ giving the radial displacement

$$
\Upsilon(\theta, \rho):=R(\theta, \rho)-\rho
$$

Observe that, instead of using the polar coordinates, we can equivalently express $\Upsilon$ on the points of $X$ as

$$
\Upsilon(z):=\|\phi(z)\|-\|z\|
$$

Note also that the angular displacement $\Theta$ can be referred directly to the points of $X$, since $\Theta(\theta, \rho)$ is the same for any $(\theta, \rho) \in \Pi^{-1}(z)$. This allows to define $\Theta(z)$ as $\Theta(\theta, \rho)$ for $z=$ $\Pi(\theta, \rho)$. 
In some applications (for instance, to some planar maps associated to ordinary differential equations), the number $\Theta(\theta, \rho)$ represents a rotation number associated to a given trajectory departing from the point $\Pi(\theta, \rho)$. In particular, observe that any solution $(\bar{\theta}, \bar{\rho}) \in$ $\Pi^{-1}(X)$ of the system

$$
\begin{gathered}
\Theta(\theta, \rho)=2 \ell \pi, \\
\Upsilon(\theta, \rho)=0
\end{gathered}
$$

determines a fixed point $\bar{z}=(\bar{x}, \bar{y})=\Pi(\bar{\theta}, \bar{\rho}) \in X$ of the map $\phi$. Such a fixed point is "tagged" with the integer $\ell$. This is an important information associated to $\bar{z}$ in the sense that, once we have fixed $\Theta$ in order to express $\tilde{\phi}$ as in (2.3), then solutions of (2.6) for different values of $\ell \in \mathbb{Z}$ determine different fixed points of $\phi$. In other words, if $\left(\bar{\theta}_{1}, \bar{\rho}_{1}\right)$ and $\left(\bar{\theta}_{2}, \bar{\rho}_{2}\right)$ are solutions of (2.6) for $\ell=\ell_{1}$ and $\ell=\ell_{2}$, respectively, with

$$
\ell_{1} \neq \ell_{2}
$$

then

$$
\bar{z}_{1}=\Pi\left(\bar{\theta}_{1}, \bar{\rho}_{1}\right) \neq \bar{z}_{2}=\Pi\left(\bar{\theta}_{2}, \bar{\rho}_{2}\right) .
$$

In fact, if, by contradiction, $\bar{z}_{1}=\bar{z}_{2}$, then $\bar{\rho}_{1}=\bar{\rho}_{2}$ and $\bar{\theta}_{2}=\bar{\theta}_{1}+2 m \pi$ for some $m \in \mathbb{Z}$. Hence, by the $2 \pi$-periodicity of $\Theta(\cdot, \rho)$, we have

$$
2 \pi \ell_{2}=\Theta\left(\bar{\theta}_{2}, \bar{\rho}_{2}\right)=\Theta\left(\bar{\theta}_{1}+2 m \pi, \bar{\rho}_{1}\right)=\Theta\left(\bar{\theta}_{1}, \bar{\rho}_{1}\right)=2 \pi \ell_{1},
$$

a contradiction.

Conversely, one can easily check that any fixed point $w \in X$ of the map $\phi$ lifts to a discrete periodic set

$$
\Pi^{-1}(w)=\{(\bar{\theta}+2 i \pi, \bar{\rho}): i \in \mathbb{Z}\}
$$

and there exists an integer $\ell=\ell_{w}$ such that each point $(\theta, \rho) \in \Pi^{-1}(w)$ is a solution of (2.6) with the same value of $\ell$.

Looking for a solution of system (2.6), an usual assumption on the map $\tilde{\phi}$ is the socalled twist condition at the boundary, which is one of the main hypotheses of the PoincaréBirkhoff fixed point theorem. In our setting, the twist condition is expressed as follows.

Definition 2.1. We say that $\tilde{\phi}$ satisfies the twist condition if

$$
\begin{array}{ll}
\Theta(\theta, \rho)<2 j \pi, & \text { for }(\theta, \rho) \in J_{i}, \\
\Theta(\theta, \rho)>2 j \pi, & \text { for }(\theta, \rho) \in J_{o}
\end{array}
$$

(or vice versa), for some $j \in \mathbb{Z}$. 
If we prefer to express the twist condition directly on $\phi$, we will write

$$
\Theta<2 j \pi \quad \text { on } X_{i}, \quad \Theta>2 j \pi \text { on } X_{o}
$$

(or viceversa).

The celebrated Poincaré-Birkhoff "twist" theorem, in its original formulation, considers the case of a standard annulus

$$
X=A[a, b], \quad X_{i}=a S^{1}, \quad X_{o}=b S^{1}, \quad 0<a<b .
$$

Then we have the following.

Theorem 2.2. Assume (2.13), and suppose that $\phi: X \rightarrow \phi(X)=X$ is an area-preserving homeomorphism which lifts on $\Pi^{-1}(X)=\mathbb{R} \times[a, b]$ to a homeomorphism $\tilde{\phi}$ of the form (2.3) which satisfies the following hypotheses:

Boundary invariance: $R(\theta, a)=a, R(\theta, b)=b$, for all $\theta \in \mathbb{R}$;

Twist condition (2.11) (for some $j \in \mathbb{Z}$ ).

Then, there exist at least two fixed points $z_{1}, z_{2}$ for $\phi$, in the interior of the annulus $X$, with $\Theta\left(z_{1}\right)=$ $\Theta\left(z_{2}\right)=2 j \pi$.

Usually, the hypothesis that the homeomorphism $\phi$ lifts to a $\tilde{\phi}$ of the form (2.3) is equivalently expressed by the assumption that $\phi$ is orientation preserving. One can easily modify the covering projection, for instance, to

$$
\Pi(\theta, \rho)=(\sqrt{2 \rho} \cos \theta, \sqrt{2 \rho} \sin \theta)
$$

in order to have that the area-preserving homeomorphism $\phi$ lifts to a homeomorphism which preserves the element of area $d \theta d \rho$.

In order to introduce the concept of bend-twist maps we recall a (wrong) attempt of proving Theorem 2.2, as described by Wilson in a letter to Birkhoff [16]

"Won't you bother with finding out what ridiculous error there is in this simple thing that occurred to me yesterday? [...] The set of the points of the annulus with $\varphi^{\prime}-\varphi=0$ may be of great complexity containing ovals or ovals within ovals in the ring. But, as this set is closed and cannot be traversed by any continuous curve from the inner to the outer circles without being cut in at least one point, such set must include at least one continuous curve circling around the ring. [...] Now, upon this curve, the shift $r^{\prime}-r$ is continuous and could not be always positive or always negative without shrinking said curve or expanding it, contrary to the supposed invariance of areas or integrals. Hence, there must be at least two points for which $r^{\prime}=r$ as well as $\varphi^{\prime}=\varphi . "$

We put in Italic the original words by Wilson. The notation is the original one and to make it compatible with that of the present paper we have to notice that the lifting of $\phi$ considered in [16] is expressed as a map $(r, \varphi) \mapsto\left(r^{\prime}, \varphi^{\prime}\right)$. Thus our condition $\Theta=0$ 


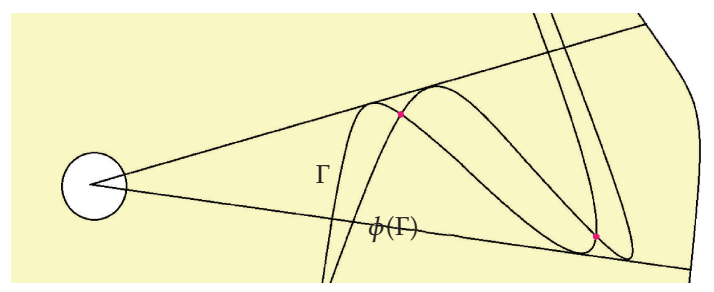

Figure 1: A sketch of the problem in Wilson's argument. We depict a sector of an annular domain in which there is a portion of a non-star-shaped curve $\Gamma$ where $\Theta=0$. The points of $\Gamma$ are moved radially to the points of $\phi(\Gamma)$ with preservation of the area. The points in $\Gamma \cap \phi(\Gamma)$ are not fixed points for $\phi$. A similar situation is described by Martins and Ureña in [6, Figures 1-2].

corresponds to $\varphi^{\prime}-\varphi=0$ in [16]. We also remark that the twist condition is assumed in [16] with $j=0$ (like in the original version of Poincaré-Birkhoff theorem).

The gap in this argument is not only on the fact that the set of points of the annulus where $\Theta=0$ may not contain a "curve" (this perhaps is not the serious mistake), but even in the case in which there is actually a simple closed curve $\Gamma \subset A[a, b]$ included in the set where $\Theta=0$, with $\Gamma$ encircling $X_{i}$, the points of $\phi(\Gamma) \cap \Gamma$ (which are supposed to exist by the area-preserving assumption) are not necessarily fixed points of $\phi$. In fact, if $\Gamma$ is not star shaped, one could well have that $\Upsilon>0$ (or $\Upsilon<0$ ) along $\Gamma$ and, at the same time, $\phi(\Gamma) \cap \Gamma \neq \emptyset$ (see Figure 1).

Of course, if we were able to prove that the radial displacement function $\Upsilon$ vanishes at some points of the locus $\Theta=0$, then we would find fixed points for $\phi$ (making the above wrong argument meaningful). From this point of view, the study of the structure of the sets of points where $\Theta=0$ may give useful information for the search of fixed points of $\phi$. Such approach was considered, for instance, by Morris in [17] who proved the existence of infinitely many periodic solutions of minimal period $2 m \pi$ (for each positive integer $m$ ), for the forced superlinear equation

$$
\ddot{x}+2 x^{3}=p(t),
$$

where $p(t)$ is a smooth function with least period $2 \pi$ and mean value zero. For his proof, Morris considered the problem of existence of fixed points for the area-preserving homeomorphism of the plane

$$
T^{m}:(a, b) \longrightarrow\left(a^{\prime}, b^{\prime}\right)=(x(2 m \pi ; a, b), \dot{x}(2 m \pi ; a, b)),
$$

where $x(t ; a, b)$ is the solution of the differential equation such that

$$
x(0 ; a, b)=a, \quad \dot{x}(0 ; a, b)=b .
$$

In [17], starlike Jordan curves around the origin $C$ were constructed such that each point $P \in C$ is mapped to $T^{m} P$ on the same ray $O P$ (see also [18] for a description of Morris result in comparison to other different approaches).

In [8] Ding considers the case of a topological annulus $\mathcal{A}$ embedded in the plane having as its boundaries two simple closed curves which are starlike with respect to the 
origin. It is assumed that there exists an analytic function $f: \mathcal{A} \rightarrow \mathcal{A}^{*}$, with $\mathcal{A}^{*}$ another starlike annulus with $\mathscr{A} \subset \mathscr{A}^{*}$ and, moreover, that $f$ satisfies the twist condition (2.11). It is also observed that the set $\Omega_{f}$ of the points in $\mathcal{A}$ where $\Theta=2 j \pi$ contains at least a Jordan curve $\Gamma$ which is not contractible in $\mathcal{A}$. The function $f$ is called a bend-twist map if there exists a Jordan curve $\Gamma \subset \Omega_{f}$, with $\Gamma$ noncontractible in $\mathcal{A}$, such that $\Upsilon$ changes its sign on $\Gamma$. Then, the following theorem holds (see [8, Theorem 7.2, page 188]).

Theorem 2.3. Let $f: \mathcal{A} \rightarrow \mathcal{A}^{*}$ be an analytic bend-twist map. Then it has at least two distinct fixed points in $A$.

We notice that, in Ding's theorem, the assumptions that $f$ is area preserving and leaves the annulus invariant are not needed. This represents a strong improvement of the hypotheses required for the Poincaré-Birkhoff twist theorem. On the other hand, the assumption that a given function is a bend-twist map does not seem easy to be checked in the applications. For this purpose, the following corollary (see [8, Corollary 7.3, page 188]) provides more explicit conditions for the applicability of the abstract result.

Corollary 2.4. Let $f: \mathcal{A} \rightarrow \mathcal{A}^{*}$ be an analytic twist map. If there are two disjoint continuous curves $\Gamma_{1}$ and $\Gamma_{2}$ in $\mathcal{A}$, connecting, respectively, the inner and the outer boundaries of $\mathcal{A}$ and such that $\Upsilon<0$ on $\Gamma_{1}$ and $\Upsilon>0$ on $\Gamma_{2}$, then $f$ is a bend-twist map on $A$, and therefore it has at least two distinct fixed points.

Our aim now is to reformulate the above results in a general topological setting in order to obtain a version of Theorem 2.3 and Corollary 2.4 for general (not necessarily analytic) maps.

Let $X \subset \mathbb{R}^{2}$ be a topological annulus (embedded in the plane) with $0 \in D(X)$, and let $\phi: X \rightarrow \mathbb{R}^{2} \backslash\{0\}$ be a continuous map admitting a lifting of the form (2.3). Let us introduce the set

$$
\Omega_{\phi}^{j}:=\{(\rho \cos \theta, \rho \sin \theta): \Theta(\theta, \rho)=2 j \pi\} .
$$

Lemma 2.5. Let $\phi$ satisfy the twist condition (2.11) for some $j \in \mathbb{Z}$. Then the set $\Omega_{\phi}^{j}$ contains a compact connected set $C^{j}$ which is essentially embedded in $X$ and $C^{j}: X_{i} \nmid X_{o}$.

Proof. Our claim is an immediate consequence of Lemma 1.4 once that we have checked that $\Omega_{\phi}^{j}: X_{i} \nmid X_{o}$. This latter property follows from the continuity of $\Theta$ and the twist condition. Indeed, if $\gamma:[0,1] \rightarrow X$ is a path with $\gamma(0) \in X_{i}$ and $\gamma(1) \in X_{o}$, then $\Theta(\gamma(t))-2 j \pi$ must vanish somewhere.

Our result corresponds to [8, Lemma 7.2, page 185] for a general $\phi$. The Jordan curve $\Gamma \subset \Omega_{f}$ considered in [8] in the analytic case is now replaced by our essentially embedded continuum $C^{j} \subset \Omega_{\phi}^{j}$. Following [8] we can now give the next definition.

Definition 2.6. Let $\phi: X \rightarrow \mathbb{R}^{2} \backslash\{0\}$ be a continuous map (admitting a lifting of the form (2.3)) which satisfies the twist condition (2.11), for some $j \in \mathbb{Z}$. We say that $\phi$ is a bend-twist map in $X$ if there exists a compact connected set $C^{j} \subset \Omega_{\phi}^{j}$ with $C^{j}$ essentially embedded in $X$ and such that $\Upsilon$ changes its sign on $C^{j}$. 
As a consequence of this definition, the following theorem, which is a version of Theorem 2.3 for mappings which are not necessarily analytic, holds.

Theorem 2.7. Let $\phi: X \rightarrow \mathbb{R}^{2} \backslash\{0\}$ be a bend-twist map. Then it has a fixed point in int $X$ and with $\Theta=2 j \pi$.

The proof is an obvious consequence of the connectedness of $C^{j}$. If we were able to prove that $C^{j}$ is a Jordan curve, then, like in [8], the existence of at least two fixed points could be ensured.

In general, and in contrast with Theorem 2.3, we cannot hope to have more than one fixed point as shown by the following example which refers to a standard planar annulus $X=A[a, b]$.

Example 2.8. Let $c:=(a+b) / 2$, and consider the set

$$
C:=\left\{(\theta, \rho): \rho=c+\varepsilon_{1} \sin ^{2}\left(\frac{\theta}{2}\right)\right\} \cup\{(2 k \pi, \rho): \rho \in[c-\delta, c+\delta], k \in \mathbb{Z}\},
$$

with $0<\varepsilon_{1}, \delta<(b-a) / 4$. The angular map $\Theta$ in $\Pi^{-1}(X)$ is defined as

$$
\Theta(\theta, \rho):= \begin{cases}-\frac{\operatorname{dist}(z, C)}{\operatorname{dist}(z, C)+\operatorname{dist}\left(z, J_{i}\right)}, & \text { for } z=(\theta, \rho), \text { with } \rho<c+\varepsilon_{1} \sin ^{2}\left(\frac{\theta}{2}\right), \\ \frac{\operatorname{dist}(z, C)}{\operatorname{dist}(z, C)+\operatorname{dist}\left(z, J_{o}\right)}, & \text { for } z=(\theta, \rho), \text { with } \rho \geq c+\varepsilon_{1} \sin ^{2}\left(\frac{\theta}{2}\right),\end{cases}
$$

while, for the radial map $R$, we set

$$
R(\theta, \rho):=\rho+\varepsilon_{2}(\rho-a)(\rho-c)(\rho-b)
$$

with $\varepsilon_{2}>0$ and sufficiently small in order to have $a \leq R(\theta, \rho) \leq b$, for all $(\theta, \rho)$. The functions $\Theta$ and $R$ define by (2.3) a continuous map $\tilde{\phi}: \mathbb{R} \times[a, b] \rightarrow \mathbb{R} \times[a, b]$ and, projecting by $\Pi$, a map $\phi: A[a, b] \rightarrow A[a, b]$. It is easy to check that $\phi$ leaves the boundaries of the annulus invariant and satisfies the twist condition (2.11) with $j=0$. The set $\Omega_{\phi}^{0}$ is the image of $C$ through $\Pi$. In accordance with Lemma 2.5 we can take $C^{0}:=\Omega_{\phi}^{0}$. The function $\Upsilon$ vanishes on the circumferences $\rho=a, \rho=b$, and $\rho=c$, and, moreover, it is negative on the open annulus $A(a, c)$ and positive on $A(c, b)$. Hence it changes its sign on $C^{0}$. However, $\phi$ has a unique fixed point in $A[a, b]$ which is $F:=(c, 0)$ (see Figure 2).

Perhaps the set $C^{0}$ in Example 2.8 is not completely satisfactory. Indeed, although it represents a compact connected set which cuts all the paths between $X_{i}$ and $X_{o}$, it is not minimal. One could suppose that if we modify Definition 2.6 by considering only minimal compact subsets of $\Omega_{\phi}^{j}$ which are essentially embedded in X, then we could provide the existence of at least two fixed points for $\phi$, like in Ding's theorem (see [9]). We have preferred to give a definition avoiding the concept of minimality because the existence of minimal sets will be only guaranteed by Zorn's lemma, and, moreover, such sets could be 


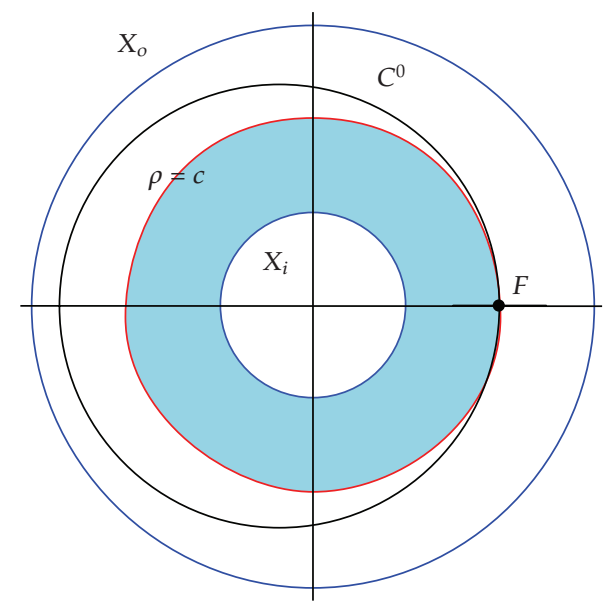

Figure 2: A description of the geometry in Example 2.8. The set $C^{0}$ made of the points of the annulus $A[a, b]$, where $\Theta=0$, is the union of a closed curve (contained in the part of the annulus between $\rho=c$ and $X_{o}$ ) and a small segment $[c-\delta, c+\delta] \times\{0\}$. The function $\Upsilon$ vanishes at $\rho=a, c, b$, and it is negative for $a<\rho<c$ and positive for $c<\rho<b$ (we have painted with a darker color the part of the annulus, where $\Upsilon<0)$. The point $F$ is the unique fixed point of $\phi$ since $\{F\}=C^{0} \cap\{\Upsilon=0\}$.

quite pathological and thus intractable from the point of view of the applications. Further investigations should be needed in this direction.

On the other hand, we are able to recover the existence of two fixed points, as in Corollary 2.4 , by the following result. In the proof we call a generalized rectangle any set which is homeomorphic to the unit square $[0,1]^{2}$.

Theorem 2.9. Let $\phi: X \rightarrow \mathbb{R}^{2} \backslash\{0\}$ be a continuous map (admitting a lifting of the form (2.3)) which satisfies the twist condition (2.11), for some $j \in \mathbb{Z}$. If there are two disjoint $\operatorname{arcs} \Gamma_{1}$ and $\Gamma_{2}$ in $X$, both connecting $X_{i}$ with $X_{o}$ in $X$ and such that $\Upsilon<0$ on $\Gamma_{1}$ and $\Upsilon>0$ on $\Gamma_{2}$, then $\phi$ has at least two distinct fixed points in int $\mathrm{X}$ with $\Theta=2 j \pi$.

Proof. Our argument is reminiscent of a similar one in the proof of the bifurcation result in [19]. Without loss of generality (up to a homeomorphism), we can suppose that $X=A[a, b]$. We also suppose (passing possibly to a sub-arc) that each $\Gamma_{n}$ intersects $X_{i}$ and $X_{o}$ exactly in one point, respectively. Let also $P_{a}^{n}$ and $P_{b}^{n}$ be the intersection points of $\Gamma_{n}$ with the circumferences $\rho=a$ and $\rho=b$, respectively, (for $n=1,2$ ). Let $C_{a}^{\prime}$ be the arc of $X_{i}$ from $P_{a}^{1}$ to $P_{a}^{2}$, and let $C_{a}^{\prime \prime}$ be the arc of $X_{i}$ from $P_{a}^{2}$ to $P_{a}^{1}$ (in the counterclockwise sense). Similarly (again in the counterclockwise sense), we determine two arcs $C_{b}^{\prime}$ and $C_{b}^{\prime \prime}$ on $X_{o}$. The Jordan curves obtained by joining $C_{a}^{\prime}, \Gamma_{2}, C_{b}^{\prime}, \Gamma_{1}$ and $C_{a}^{\prime \prime}, \Gamma_{1}, C_{b}^{\prime \prime}, \Gamma_{2}$ bound two generalized rectangles $\mathcal{R}_{1}$ and $\boldsymbol{R}_{2}$. We claim that in the interior of $\mathcal{R}_{n}(n=1,2)$ there exists at least one fixed point for $\phi$ having $j$ as associated rotation number. We prove the claim for $\mathcal{R}_{1}$, since the proof for $\mathcal{R}_{2}$ is exactly the same.

First of all, by the covering projection $\Pi$, we lift the set $R_{1}$ to the strip

$$
\Pi^{-1}(X)=\mathbb{R} \times[a, b]
$$


and observe that $\Pi^{-1}\left(\mathcal{R}_{1}\right)$ can be written as

$$
\Pi^{-1}\left(\mathcal{R}_{1}\right)=\boldsymbol{R}+(2 m \pi, 0),
$$

with $\boldsymbol{R}$ a generalized rectangle contained in the strip and such that its boundary projects homeomorphically onto $\partial \mathcal{R}_{1}$ by $\Pi$. As observed above, $\mathcal{R}_{1}$ is the compact region of the plane bounded by the Jordan curve $C_{a}^{\prime}, \Gamma_{2}, C_{b}^{\prime}, \Gamma_{1}$. By the Schoenflies theorem [10] we can choose a homeomorphism $\eta:[0,1]^{2} \rightarrow \boldsymbol{R}$ in such a manner that

$$
\begin{aligned}
& (\Pi \circ \eta)([0,1] \times\{0\})=C_{a}^{\prime}, \quad(\Pi \circ \eta)([0,1] \times\{1\})=C_{b^{\prime}}^{\prime} \\
& (\Pi \circ \eta)(\{0\} \times[0,1])=\Gamma_{2}, \quad(\Pi \circ \eta)(\{1\} \times[0,1])=\Gamma_{1} .
\end{aligned}
$$

The vector field

$$
f=\left(f_{1}, f_{2}\right):[0,1]^{2} \longrightarrow \mathbb{R}^{2},
$$

defined by

$$
f(x, y):=(\Upsilon(\eta(x, y)), \Theta(\eta(x, y))-2 j \pi),
$$

is such that

$$
\begin{gathered}
f_{1}(0, y)=\Upsilon(\theta, \rho) \quad \text { with } \Pi(\theta, \rho) \in \Gamma_{2}, \forall y \in[0,1], \\
f_{1}(1, y)=\Upsilon(\theta, \rho) \quad \text { with } \Pi(\theta, \rho) \in \Gamma_{1}, \forall y \in[0,1], \\
f_{2}(x, 0)=\Theta(\theta, \rho)-2 j \pi \quad \text { with } \Pi(\theta, \rho) \in C_{a}^{\prime}, \forall x \in[0,1], \\
f_{2}(x, 1)=\Theta(\theta, \rho)-2 j \pi \quad \text { with } \Pi(\theta, \rho) \in C_{b}^{\prime}, \forall x \in[0,1] .
\end{gathered}
$$

Thus, by the assumptions on $\Theta$ and $\Upsilon$, we find that

$$
\begin{aligned}
& f_{1}(0, y)>0>f_{1}(1, y), \quad \forall y \in[0,1] \\
& f_{2}(x, 0)<0<f_{2}(x, 1), \quad \forall x \in[0,1] .
\end{aligned}
$$

The above (strict) inequalities imply that we are in the setting of a two-dimensional version of the Poincaré-Miranda theorem and that

$$
\operatorname{deg}(f,] 0,1\left[{ }^{2}, 0\right)=-1,
$$

where "deg" denotes the Brouwer degree. Therefore there exists at least one point $\left(x^{*}, y^{*}\right)$ such that $f\left(x^{*}, y^{*}\right)=0$. This, in turns, implies the existence of a fixed point $\left(\theta^{*}, \rho^{*}\right)=$ $\eta\left(x^{*}, y^{*}\right) \in$ int $\mathcal{R}$ such that $\Pi\left(\theta^{*}, \rho^{*}\right)$ is a fixed point of $\phi$ in the interior of $\mathcal{R}_{1}$ and such that $\Theta=2 j \pi$.

With the same argument of the proof of Theorem 2.9, the next result can be obtained. 
Corollary 2.10. Let $\phi: X \rightarrow \mathbb{R}^{2} \backslash\{0\}$ be a continuous map (admitting a lifting of the form (2.3)) which satisfies the twist condition (2.11), for some $j \in \mathbb{Z}$. Assume that there exist $2 k$ disjoint arcs $(k \geq$ 1) connecting $X_{i}$ with $X_{o}$ in $X$. We label these arcs in a cyclic order $\Gamma_{1}, \Gamma_{2}, \ldots, \Gamma_{n}, \ldots, \Gamma_{2 k}, \Gamma_{2 k+1}=\Gamma_{1}$ and assume that $\Upsilon<0$ on $\Gamma_{n}$ for $n$ odd and $\Upsilon>0$ on $\Gamma_{n}$ for $n$ even (or viceversa). Then $\phi$ has at least $2 k$ distinct fixed points in int $X$, all the fixed points with $\Theta=2 j \pi$.

Remark 2.11. Observe that Theorem 2.9, as well as Corollary 2.10, is stable with respect to small continuous perturbations of the map $\phi$. This follows from the fact that (2.29) is true for any function $f$ satisfying the strict inequalities (2.28). Thus, if we perturb the function $\phi$ with a new continuous map $\psi$ with $\|\psi-\phi\|_{\infty} \leq \varepsilon$ on $X$ and $\varepsilon>0$ sufficiently small, we have that the twist condition and the conditions on $\Upsilon$ on $\Gamma_{1}$ and $\Gamma_{2}$ are satisfied also for $\psi$, and hence we get fixed points for $\psi$ as well.

On the other hand, we remark that Theorem 2.7 as well as Theorem 2.3 is not stable even in case of arbitrarily small perturbations. In order to show this fact, let us consider the following example.

Example 2.12. Let $X:=A[a, b]$ be a planar annulus with $a=1 / 2$ and $b=5$. We consider an angular map $\Theta$ in $\Pi^{-1}(X)$ as $\Theta(\theta, \rho):=(\rho-3)^{2}(\rho-1)$, while, for the radial map, we take $R(\theta, \rho):=\rho+\rho^{2}\left(\cos ^{2} \theta+4 \sin ^{2} \theta\right)-16$. The functions $\Theta$ and $R$ define by (2.3) a continuous $\operatorname{map} \tilde{\phi}: \mathbb{R} \times[a, b] \rightarrow \mathbb{R} \times[a, b]$ and, projecting by $\Pi$, a map $\phi: A[a, b] \rightarrow A[a, b]$. It is easy to check that $\phi$ satisfies the twist condition (2.11) with $j=0$. The set $\Omega_{\phi}^{0}$ is the union of the circumferences $S^{1}$ and $3 S^{1}$. The function $\Upsilon$ vanishes on the ellipse $x^{2}+4 y^{2}=16$. According to Definition 2.6, the map $\phi$ is a bend-twist map as $\Upsilon$ changes its sign on $3 S^{1}$. Indeed $\phi$ has exactly four fixed points which are the intersections of the ellipse with the circumference $3 S^{1}$. However, for any $\varepsilon>0$ sufficiently small, the map $\phi_{\varepsilon}:=M_{\varepsilon} \circ \phi$ (where $M_{\varepsilon} z:=z \exp (i \varepsilon$ ) is a rotation of a small angle $\varepsilon$ ) has no fixed points in $X$. The reason is that the set $3 S^{1}$ disappears after an arbitrary small perturbation for $\varepsilon>0$, while the set $S^{1}$ is stable (in the sense that it continues into a nearby closed Jordan curve) but it is not suitable for the bend-twist map theorem since $\Upsilon$ has constant sign on it.

Up to now we have presented all our results in terms of liftings of planar maps given by the standard covering projection $\Pi$ in polar coordinates. In this manner we could make a simpler comparison with other results, like the Poincaré-Birkhoff fixed point theorem and the Ding analytic bend-twist maps theorem, which are usually expressed in the same framework. It is clear, however, that our approach works exactly the same also if different covering projections are used. For instance, in the applications to planar systems which are a perturbation of the first-order Hamiltonian system

$$
\begin{gathered}
\dot{x}=\frac{\partial H}{\partial y}(x, y), \\
\dot{y}=-\frac{\partial H}{\partial x}(x, y),
\end{gathered}
$$

if we have an annulus filled by periodic orbits of (2.30), it could be convenient to choose as a radial coordinate the number $\mathcal{\varepsilon}$ expressing the level of the Hamiltonian and as an angular coordinate a normalized time of the corresponding orbit at level $\mathcal{\varepsilon}$. We are going to use this remark for the application in the next section (see $[19,20]$ for some analogous cases). 


\section{An Application}

It appears that the presence of bend-twist maps associated to planar differential equations is ubiquitous. This does not mean that proving their existence in concrete equations would be a simple task. It is a common belief that periodic solutions obtained for planar Hamiltonian systems via the Poincaré-Birkhoff fixed point theorem are not preserved by arbitrarily small perturbations which destroy the Hamiltonian structure of the equations. A typical example occurs when we add a small friction to a conservative system of the form

$$
\ddot{x}+f(x)=0,
$$

passing to

$$
\ddot{x}+\varepsilon \dot{x}+f(x)=0 .
$$

In general, for any continuous $f$ and each continuous function $\delta: \mathbb{R} \rightarrow \mathbb{R}$ such that $\delta(s) s>0$ for all $s \neq 0$, the only possible periodic solutions of

$$
\ddot{x}+\delta(\dot{x})+f(x)=0
$$

are the constant ones, corresponding to the zeros of $f$ (if any).

For (3.1) one can easily find conditions on $f(x)$ guaranteeing the existence of an annulus in the phase plane filled by periodic orbits of the equivalent first-order Hamiltonian system

$$
\begin{gathered}
\dot{x}=y, \\
\dot{y}=-f(x) .
\end{gathered}
$$

To present a specific example, let us assume that there exists an open interval $I:=] a, b[$ with $-\infty \leq a<0<b \leq+\infty$ such that $f: I \rightarrow \mathbb{R}$ is locally Lipschitz continuous with $f(0)=0$ and

$$
f(s) s>0, \quad \forall s \in I \backslash\{0\} .
$$

The corresponding potential function

$$
F(x):=\int_{0}^{x} f(s) d s
$$

is strictly decreasing on $] a, 0]$ and strictly increasing on $[0, b[$. Hence, for every constant $c$ with

$$
0<c<C:=\min \left\{F\left(a^{+}\right), F\left(b^{-}\right)\right\},
$$

the energy level line $E^{c}$ defined by

$$
E(x, y)=c, \quad \text { for } E(x, y):=\frac{1}{2} y^{2}+F(x), x \in I,
$$


is a closed periodic orbit surrounding the origin. We denote by $\tau_{c}$ the fundamental period of $E^{c}$. By the above assumptions it turns out that the map $c \mapsto \tau_{c}$ is continuous (see, for instance, [19, (v) page 83], where such result is proved in a more general situation).

In this setting we propose an application of the Poincaré-Birkhoff twist theorem and the bend-twist maps theorem to equations which are small perturbations of (3.1).

Just to start, we suppose that there exist $c_{1}$ and $c_{2}$ such that

$$
\tau_{1}<\tau_{2}
$$

for $\tau_{i}:=\tau_{c_{i}}$. For convenience in the next exposition, we also suppose that

$$
0<c_{1}<c_{2}<C
$$

The case in which $c_{2}<c_{1}$ can be treated analogously. The planar annulus

$$
\mathcal{A}:=\left\{(x, y) \in I \times \mathbb{R}: c_{1} \leq E(x, y) \leq c_{2}\right\}
$$

is filled by periodic trajectories whose period varies continuously with the parameter $c$. In particular the inner boundary $\mathcal{A}_{i}$ and the outer boundary $\mathcal{A}_{0}$ of the annulus are the energy level lines $E^{c_{1}}$ and $E^{c_{2}}$, respectively.

Consider the level line $E^{c}$ with $c_{1} \leq c \leq c_{2}$. By (3.5) it follows that $E^{c}$ is strictly star shaped around the origin. Hence, for every angle $\theta$, the line

$$
L_{\theta}:=\{(\rho \cos \theta, \rho \sin \theta): \rho>0\}
$$

intersects $E^{c}$ exactly in one point. From this fact, we immediately obtain another covering projection map onto the annulus which is equivalent to the projection in polar coordinates $\Pi$. In this manner, we can describe the points of $\mathcal{A}$ by means of pairs $(\theta, \varepsilon)$, where, for each point $z \in \mathcal{A}$, we have that $\theta$ is the usual angle in polar coordinates and $\varepsilon=E(z)$.

The continuity of the map $c \mapsto \tau_{c}$ implies that, for every $T$ with

$$
\tau_{1}<T<\tau_{2}
$$

equation (3.1) has at least one $T$-periodic solution $\widehat{x}(\cdot)$, where, for

$$
\widehat{c}:=E(\widehat{x}(0), \dot{\hat{x}}(0)),
$$

we have that $\tau_{\widehat{c}}=T$. Actually, due to the autonomous nature of the system, there is at least a continuum of periodic solutions given by the shifts in time of $\hat{x}$, that is, the functions $\hat{x}_{\theta}(\cdot)$, with $\widehat{x}_{\theta}(t)=\widehat{x}(t+\theta)$. From the point of view of the Poincare map, which is the map

$$
\Phi: z \longmapsto \phi(T, z),
$$


where $\phi(\cdot, z)$ is the solution of (3.4) with $\phi(0, z)=z$, we have that $\Phi$ has a continuum of fixed points which are all the points of the closed curve $E^{\widehat{c}}$. The uniqueness of the periodic trajectory is not guaranteed (unless we assume some further conditions, like the strict monotonicity of the period with respect to $c$ ). In this autonomous case, as we have observed above, an arbitrarily small perturbation destroying the Hamiltonian structure of the equation may have the effect that the nontrivial $T$-periodic solutions disappear.

As a next step, we consider a perturbation of (3.1) in the form of

$$
\ddot{x}+(1+w(t)) f(x)=0,
$$

where $w: \mathbb{R} \rightarrow \mathbb{R}$ is a $T$-periodic function. For our purposes, only weak regularity assumptions on $w(\cdot)$ are needed. For instance, we can suppose that $w \in L^{1}(0, T)$ and consider the solutions of (3.16) in the generalized (Carathéodory) sense (see [21]). In this case, by the theorem of continuous dependence of the solutions in the Carathéodory setting, the Poincaré map associated to the planar system

$$
\begin{gathered}
\dot{x}=y, \\
\dot{y}=-(1+w(t)) f(x)
\end{gathered}
$$

is well defined on $A$ if $w(t)$ is sufficiently small in the $L^{1}$-norm on $[0, T]$. Then the following theorem holds.

Theorem 3.1. Assume (3.13). Then there exists $\epsilon>0$ such that for each $w(\cdot)$ with $|w|_{L^{1}(0, T)}<\epsilon$ (3.16) has at least two T-periodic solutions with initial value in $\mathcal{A}$, for $\mathbb{A}$ defined in (3.11).

Theorem 3.1 is substantially a variant of a result of Buttazzoni and Fonda [22]. The proof follows a version of the Poincare-Birkhoff fixed point theorem due to Ding [23] which applies to an area-preserving twist homeomorphism of a planar annulus with star-shaped boundaries. To be more precise, it should be remarked that recently the counterexamples in $[6,7]$ have shown that the theorem fails for annular domains with non-star-shaped boundaries. Here we use a result by Rebelo [14, Corollary 2] which holds for an areapreserving homeomorphism of the plane $\Psi$ such that $\Psi(0)=0$ and with $\Psi$ satisfying a twist condition on the boundary of a starlike annulus surrounding the origin.

We give a sketch of the proof of Theorem 3.1 for the reader's convenience.

Proof. If we denote by $\psi(\cdot, z)=\left(\psi_{1}(\cdot, z), \psi_{2}(\cdot, z)\right)$ the solution of $(3.17)$ with $\psi(0, z)=z$ and by $\Psi$ the corresponding Poincaré map

$$
\Psi(z):=\psi(T, z)
$$

we have that $\Psi$ is defined on

$$
\Phi:=\left\{(x, y) \in I \times \mathbb{R}: E(x, y) \leq c_{2}\right\}
$$


(if $|w|_{L^{1}(0, T)}$ is sufficiently small) as an area-preserving homeomorphism of $\boldsymbol{\Phi}$ onto $\Psi(\boldsymbol{\Phi})$ with $\Psi(0)=0$ and $\psi(t, z) \neq 0$, for all $t \in[0, T]$ and $z \in \mathcal{A}$. Passing to the polar coordinates we can determine an angular function $\vartheta(t, z)$ so that

$$
\psi(t, z)=\|\psi(t, z)\|(\cos (\vartheta(t, z)), \sin (\vartheta(t, z))) .
$$

It turns out that, in terms of the lifting $\widetilde{\Psi}$ associated to $\Psi$ (compare to (2.3)), we have that

$$
\widetilde{\Psi}(\theta, \rho)=(\theta+\Theta(\theta, \rho), R(\theta, \rho))
$$

with

$$
\begin{gathered}
R(\theta, \rho)=\|\Psi(z)\| \\
\Theta(\theta, \rho)=\vartheta(0, z)-\vartheta(T, z)=\int_{0}^{T} \frac{(1+w(t)) f\left(\psi_{1}(t, z)\right) \psi_{1}(t, z)+\psi_{2}^{2}(t, z)}{\|\psi(t, z)\|^{2}} d t
\end{gathered}
$$

for $z=(\rho \cos \theta, \rho \sin \theta)$ (see [24] for the details). Assumption (3.13) for system (3.4) which now is viewed as a comparison system for (3.17) implies that if the perturbation $w(\cdot)$ is sufficiently small, then $\Theta>2 \pi$ on $\mathcal{A}_{i}$ and $\Theta<2 \pi$ on $\mathcal{A}_{0}$ and thus the twist condition (2.11) holds for $j=1$.

Finally, using the fact that $\mathcal{A}_{i}$ and $\mathcal{A}_{0}$ are strictly star shaped with respect to the origin with $\Psi(0)=0$, we can apply Ding's version of the Poincare-Birkhoff theorem [14, 23] and the existence of at least two distinct fixed points for $\Psi$ in the interior of $\mathscr{A}$ is ensured.

A natural question that now can arise is whether such (nontrivial) $T$-periodic solutions would persist if a sufficiently small perturbation which destroys the Hamiltonian structure of the equation is performed. In the abstract setting of the Poincaré-Birkhoff theorem an answer can be found in the papers by Neumann [25] and Franks [26, 27] according to which if we have a finite number of fixed points then there are also fixed points with nonzero index. Actually, in [25, Theorem 2.1], the more general situation that the set of fixed points does not separate the boundaries is considered as well. In such cases, a standard application of the fixed point index theory (or the topological degree theory for maps of the plane) guarantees the persistence of fixed points for maps which are close to the Poincare map, and hence the existence of nontrivial $T$-periodic solutions also for sufficiently small perturbations of (3.16) holds. From this point of view, we could say that the bend-twist map theorem, in the form of Theorem 2.9 provides an effective criterion to prove the persistence of periodic solutions under small perturbations. In order to show an example of (3.16) to which our result can be applied, we consider a special form of the $T$-periodic weight $w(t)$. For simplicity in the exposition we confine ourselves to the case of a continuous and $T$-periodic function $w: \mathbb{R} \rightarrow$ $\mathbb{R}$ such that there is an interval $] t_{0}, t_{1}[\subset[0, T]$ such that

$$
w(t)>0, \quad \forall t \in] t_{0}, t_{1}[, \quad w(t)=0, \quad \forall t \in[0, T] \backslash] t_{0}, t_{1}[
$$

By the continuity of $w(\cdot)$ we can get the following corollary of Theorem 3.1 where the smallness of $w$ in the $L^{1}$-norm is expressed in terms of $\delta_{0}$. 
Corollary 3.2. Assume (3.13), and let $w(\cdot)$ be a continuous and T-periodic function satisfying (3.23). Then there exists $\delta_{0}>0$ such that, if

$$
t_{1}-t_{0}<\delta_{0}
$$

equation (3.16) has at least two T-periodic solutions with initial value in $A$.

In comparison to this result obtained via the Poincaré-Birkhoff fixed point theorem, using Corollary 2.10 we can obtain the following.

Theorem 3.3. Assume (3.13), and let $w(\cdot)$ be a continuous and T-periodic function satisfying (3.23). Then there exists $\delta_{1}>0$ such that, if

$$
t_{1}-t_{0}<\delta_{1}
$$

equation (3.16) has at least four T-periodic solutions with initial value in A. Moreover, the result is robust with respect to small perturbations. In particular, for

$$
\ddot{x}+\varepsilon \dot{x}+(1+w(t)) f(x)=0,
$$

there are at least four T-periodic solutions with initial value in the annulus $\mathbb{A}$ if $\varepsilon$ is sufficiently small.

Proof. Without loss of generality (via a time shift leading to an equivalent equation), we can suppose that

$$
w(t)=0, \quad \forall t \in[0, T-\delta], \quad w(t)>0, \quad \forall t \in] T-\delta, T[,
$$

where we have set

$$
\delta:=t_{1}-t_{0}
$$

To begin with the proof, we consider the Poincare map $\Psi$ on the annulus $\mathbb{A}$, with $\Psi$ defined as in (3.18). Passing to the polar coordinates and following the same argument as in the proof of Theorem 3.1, we find a constant $\delta_{0}$ such that if $\delta<\delta_{0}$ then $\Theta>2 \pi$ on $A_{i}$ and $\Theta<2 \pi$ on $\mathcal{A}_{0}$ and hence the twist condition (2.11) holds for $j=1$.

In order to check the validity of the condition on the map $\Upsilon$, it is for us convenient to enter in the setting of the modified polar coordinates $(\theta, \varepsilon)$, instead of the standard polar coordinates $(\theta, \rho)$. In this case, we can express the function $\Upsilon$ as

$$
\Upsilon(z):=E(\Psi(z))-E(z)
$$

We split now the map $\Psi$ as

$$
\Psi=\Psi_{2} \circ \Psi_{1}
$$


with $\Psi_{1}$ and $\Psi_{2}$ defined as follows:

$$
\Psi_{1}(z):=\phi(T-\delta, z)
$$

where $\phi(\cdot, z)$ is the solution of the autonomous system (3.4) with $\phi(0, z)=z$ and

$$
\Psi_{2}(z):=\psi(T ; T-\delta, z)
$$

where $\psi(\cdot ; T-\delta, z)$ is the solution of system (3.17) which departs from the point $z$ at the time $T-\delta$. In this splitting we have also used the fact that system (3.17) coincides with the autonomous system (3.4) on $[0, T-\delta]$. Hence we have

$$
E\left(\Psi_{1}(z)\right)=E(z), \quad \forall z \in \mathcal{A}
$$

Let us consider now a solution $\psi(t)=\left(\psi_{1}(t), \psi_{2}(t)\right)$ of (3.17) and evaluate the energy $E$ along such solution. We obtain

$$
\begin{aligned}
\frac{d}{d t} E\left(\psi_{1}(t), \psi_{2}(t)\right) & =\psi_{2}^{\prime}(t) \psi_{2}(t)+f\left(\psi_{1}(t)\right) \psi_{1}^{\prime}(t) \\
& =-(1+w(t)) f\left(\psi_{1}(t)\right) \psi_{2}(t)+f\left(\psi_{1}(t)\right) \psi_{2}(t) \\
& =-w(t) f\left(\psi_{1}(t)\right) \psi_{2}(t) .
\end{aligned}
$$

For $t \in] T-\delta, T$ [ we have that $w(t)>0$, and therefore the energy evaluated on a solution for the time interval $[T-\delta, T]$ is decreasing as long as the solution remains in the first or in the third quadrant and is increasing as long as the solution remains in the second or in the fourth quadrant.

Let $\alpha \in] 0, \pi / 2$ [ be a fixed angle (the smaller $\alpha$ we take, the larger $\delta_{1}$ will be allowed). Recalling the definition of $L_{\theta}$ in (3.12), let $\Lambda_{1}$ be the intersection of the line $L_{\pi / 2-\alpha}$ with the annulus $A$. We are interested in the motion of the points of $\Lambda_{1}$ under the action of $\Psi_{2}$. Since $(d / d t) \vartheta(t)>0$, the points of $\Lambda_{1}$ move in the clockwise sense, and therefore they remain in the first quadrant if $\delta$ is sufficiently small. Hence $(d / d t) E\left(\psi_{1}(t), \psi_{2}(t)\right)$ is negative for $t \in[T-\delta, T]$ when $\left(\psi_{1}(0), \psi_{2}(0)\right) \in \Lambda_{1}$. This proves that

$$
E\left(\Psi_{2}(z)\right)-E(z)<0, \quad \forall z \in \Lambda_{1}
$$

Arguing in the same manner, we have that

$$
\begin{gathered}
E\left(\Psi_{2}(z)\right)-E(z)>0, \quad \forall z \in \Lambda_{2}:=L_{\pi-\alpha} \cap \mathcal{A} \\
E\left(\Psi_{2}(z)\right)-E(z)<0, \quad \forall z \in \Lambda_{3}:=L_{(3 \pi / 2)-\alpha} \cap \mathcal{A} \\
E\left(\Psi_{2}(z)\right)-E(z)>0, \quad \forall z \in \Lambda_{4}:=L_{2 \pi-\alpha} \cap \mathcal{A} .
\end{gathered}
$$

All these relations hold provided that $\delta$ is chosen suitably small (say $\delta<\delta_{1}$ ) so that the solutions of (3.17) which depart at the time $T-\delta$ from $\Lambda_{i}$, remain in the same quadrant of 
$\Lambda_{i}$ for all $t \in[T-\delta, T]$. In order to make such argument more precise we can evaluate the angular displacements and choose $\delta>0$ such that

$$
\int_{T-\delta}^{T} \frac{(1+w(t)) f\left(\psi_{1}(t, z)\right) \psi_{1}(t, z)+\psi_{2}^{2}(t, z)}{\|\psi(t, z)\|^{2}} d t<\frac{\pi}{2}-\alpha
$$

holds for all $z \in \Lambda_{i}(i=1, \ldots, 4)$.

Finally, recalling (3.33) and the definition of $\Upsilon$ in the $(\theta, \varepsilon)$-coordinates given in (3.29) and setting

$$
\Gamma_{i}:=\Psi_{1}^{-1}\left(\Lambda_{i}\right), \quad i=1, \ldots, 4,
$$

we conclude that $\Upsilon<0$ on $\Gamma_{i}$ for $i$ odd and $\Upsilon>0$ on $\Gamma_{i}$ for $i$ even. The thesis is thus achieved using Corollary 2.10.

An analysis of the proof and of inequality (3.37) shows that our argument is still valid if we take $w(t)=\chi_{\left[t_{0}, t_{1}\right]} W(t)$, where $W(\cdot)$ is a fixed positive function in $L^{1}([0, T])$.

Clearly, the same result holds also for (3.26) which can be viewed as a perturbation of (3.16). Of course, for such an application we exploit also the fact that in Corollary 2.10 no area-preserving-type hypothesis is required. The smallness of $\varepsilon$ will depend on the smallness of $\delta_{1}$.

We have achieved our result for a very special form of the weight function. A natural question concerns which kind of shape for a $T$-periodic coefficient $q(t)$ may be required in order to obtain a similar result for

$$
\ddot{x}+q(t) f(x)=0 .
$$

Generally speaking our argument may work (modulo technical difficulties) whenever we can split the behavior of the solutions of the equivalent system in the phase plane into two regimes, depending on a different shape of $q(t)$ in two subintervals of its domain. In at least one of these regimes, we need to have a control of the trajectories and prove that they do not go too far from an annular region described by the level lines of an associated autonomous system. In the other regime, we need to show that there are at least some trajectories which are, in some sense, transverse to the annulus (and move into opposite directions). A different application of our technique is contained in [9] where we have considered a model of fluid mixing which is reminiscent to the case in which $q(t)$ changes its sign.

A theorem about the existence of four solutions in this setting appears rather unusual (with respect to Corollary 3.2 and other analogous results following from the PoincaréBirkhoff twist theorem). For previous multiplicity results in a completely different setting (namely, the Floquet problem for a superlinear equation), see [28].

\section{Acknowledgments}

The authors are grateful to Professor Tongren Ding for providing them with a copy of his interesting book [8] on its publication. They also thank the referees for the careful checking of their paper and for some useful remarks. 


\section{References}

[1] H. Poincaré, "Sur un théorème de geométrie," Rendiconti del Circolo Matematico di Palermo, vol. 33, pp. 375-389, 1912.

[2] G. D. Birkhoff, "Proof of Poincaré's geometric theorem," Transactions of the American Mathematical Society, vol. 14, pp. 14-22, 1913.

[3] G. D. Birkhoff, "An extension of Poincaré's last geometric theorem," Acta Mathematica, vol. 47, no. 4, pp. 297-311, 1925.

[4] M. Brown and W. D. Neumann, "Proof of the Poincaré-Birkhoff fixed point theorem," Michigan Mathematical Journal, vol. 24, no. 1, pp. 21-31, 1977.

[5] F. Dalbono and C. Rebelo, "Poincaré-Birkhoff fixed point theorem and periodic solutions of asymptotically linear planar Hamiltonian systems," Rendiconti del Seminario Matematico Università e Politecnico di Torino, vol. 60, no. 4, pp. 233-263, 2003.

[6] R. Martins and A. J. Ureña, "The star-shaped condition on Ding's version of the Poincaré-Birkhoff theorem," Bulletin of the London Mathematical Society, vol. 39, no. 5, pp. 803-810, 2007.

[7] P. Le Calvez and J. Wang, "Some remarks on the Poincare-Birkhoff theorem," Proceedings of the American Mathematical Society, vol. 138, no. 2, pp. 703-715, 2010.

[8] T. Ding, Approaches to the Qualitative Theory of Ordinary Differential Equations, vol. 3 of Peking University Series in Mathematics, World Scientific, Hackensack, NJ, USA, 2007.

[9] A. Pascoletti and F. Zanolin, "A crossing lemma for annular regions and invariant sets," Preprint.

[10] E. E. Moise, Geometric Topology in Dimensions 2 and 3, Springer, New York, NY, USA, 1977.

[11] A. Berarducci, D. Dikranjan, and J. Pelant, "Uniform quasi components, thin spaces and compact separation," in Proceedings of the International Conference on Topology and its Applications (Yokohama, 1999), vol. 122, pp. 51-64, 2002.

[12] J. G. Hocking and G. S. Young, Topology, Dover Publications, New York, NY, USA, 2nd edition, 1988.

[13] D. E. Sanderson, "Advanced plane topology from an elementary standpoint," Mathematics Magazine, vol. 53, no. 2, pp. 81-89, 1980.

[14] C. Rebelo, "A note on the Poincaré-Birkhoff fixed point theorem and periodic solutions of planar systems," Nonlinear Analysis, vol. 29, no. 3, pp. 291-311, 1997.

[15] M. J. Greenberg, Lectures on Algebraic Topology, W. A. Benjamin Inc., New York, NY, USA, 1967.

[16] G. D. Birkhoff, "Sur la demonstration directe du renier théorème de Henri Poincaré par M. Dantzig," Bulletin des Sciences Mathématiques, vol. 42, pp. 41-43, 1918.

[17] G. R. Morris, "An infinite class of periodic solutions of $\ddot{x}+2 x^{3}=p(t)$," Mathematical Proceedings of the Cambridge Philosophical Society, vol. 61, pp. 157-164, 1965.

[18] A. Capietto, J. Mawhin, and F. Zanolin, "Periodic solutions of some superlinear functional differential equations," in Proceedings of the International Symposium on Functional Differential Equations (Kyoto, 1990), pp. 19-31, Singapore, 1991, World Scientific.

[19] M. Henrard and F. Zanolin, "Bifurcation from a periodic orbit in perturbed planar Hamiltonian systems," Journal of Mathematical Analysis and Applications, vol. 277, no. 1, pp. 79-103, 2003.

[20] A. Margheri, C. Rebelo, and F. Zanolin, "Chaos in periodically perturbed planar Hamiltonian systems using linked twist maps," Journal of Differential Equations, vol. 249, no. 12, pp. 3233-3257, 2010.

[21] J. K. Hale, Ordinary Differential Equations, Robert E. Krieger, Huntington, NY, USA, 2nd edition, 1980.

[22] P. Buttazzoni and A. Fonda, "Periodic perturbations of scalar second order differential equations," Discrete and Continuous Dynamical Systems, vol. 3, no. 3, pp. 451-455, 1997.

[23] W. Y. Ding, "A generalization of the Poincare-Birkhoff theorem," Proceedings of the American Mathematical Society, vol. 88, no. 2, pp. 341-346, 1983.

[24] F. Zanolin, "Time-maps and boundary value problems for ordinary differential equations," in Tricomi's Ideas and Contemporary Applied Mathematics (Rome/Turin, 1997), vol. 147 of Atti Convegni Lincei, pp. 281-302, Accad. Naz. Lincei, Rome, Italy, 1998.

[25] W. D. Neumann, "Generalizations of the Poincaré Birkhoff fixed point theorem," Bulletin of the Australian Mathematical Society, vol. 17, no. 3, pp. 375-389, 1977.

[26] J. Franks, "Generalizations of the Poincaré-Birkhoff theorem," Annals of Mathematics. Second Series, vol. 128 , no. 1, pp. 139-151, 1988.

[27] J. Franks, "Erratum: generalizations of the Poincaré-Birkhoff theorem," Annals of Mathematics. Second Series, vol. 164, no. 2, pp. 1097-1098, 2006.

[28] M. Henrard, "Degree for superlinear Floquet boundary value problems and existence of four solutions with a prescribed number of zeros," Report 25/97/M, SISSA, 1997. 


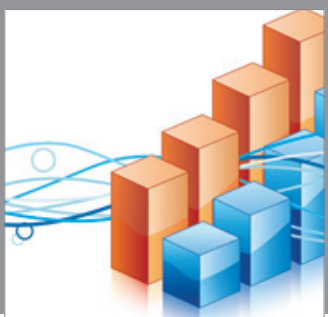

Advances in

Operations Research

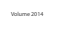

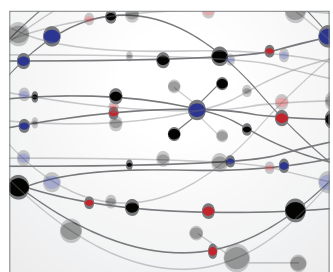

\section{The Scientific} World Journal
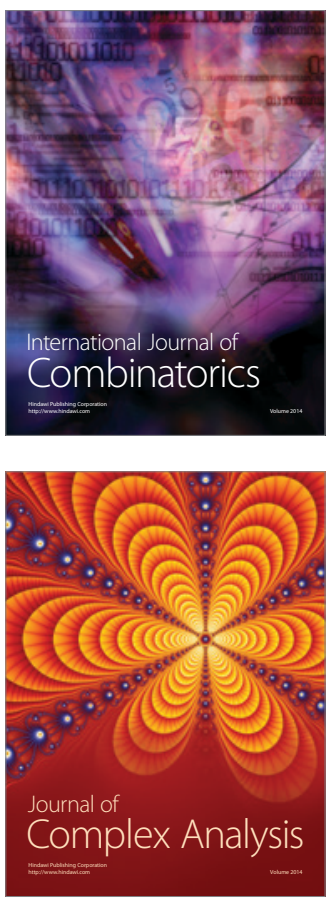

International Journal of

Mathematics and

Mathematical

Sciences
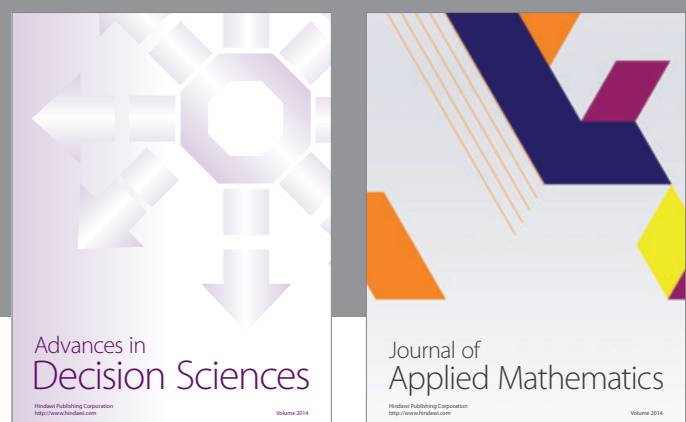

Journal of

Applied Mathematics
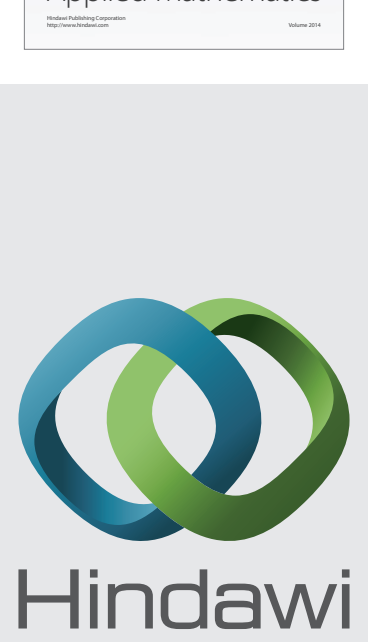

Submit your manuscripts at http://www.hindawi.com
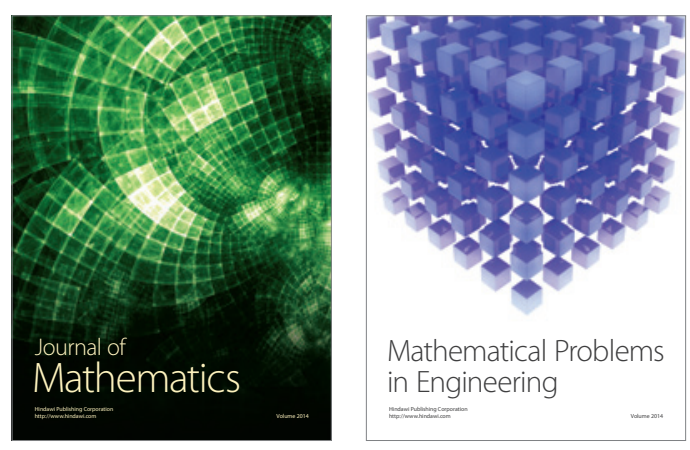

Mathematical Problems in Engineering
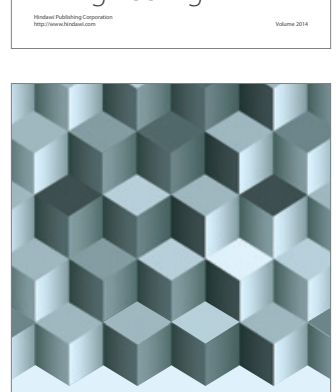

Journal of

Function Spaces
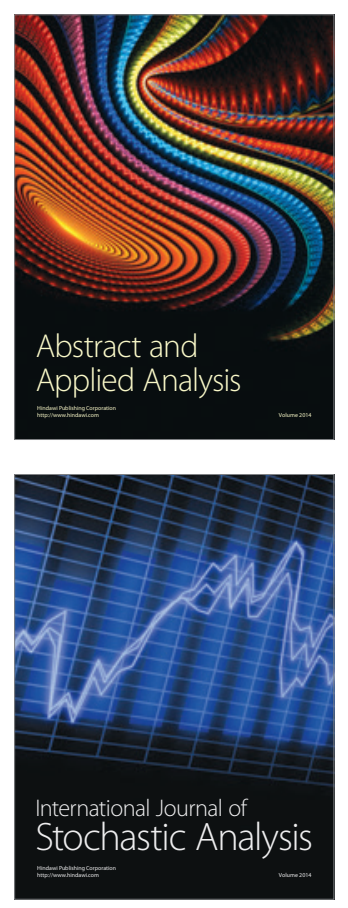

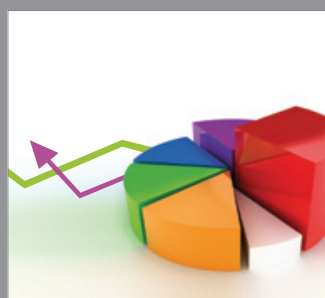

ournal of

Probability and Statistics

Promensencen
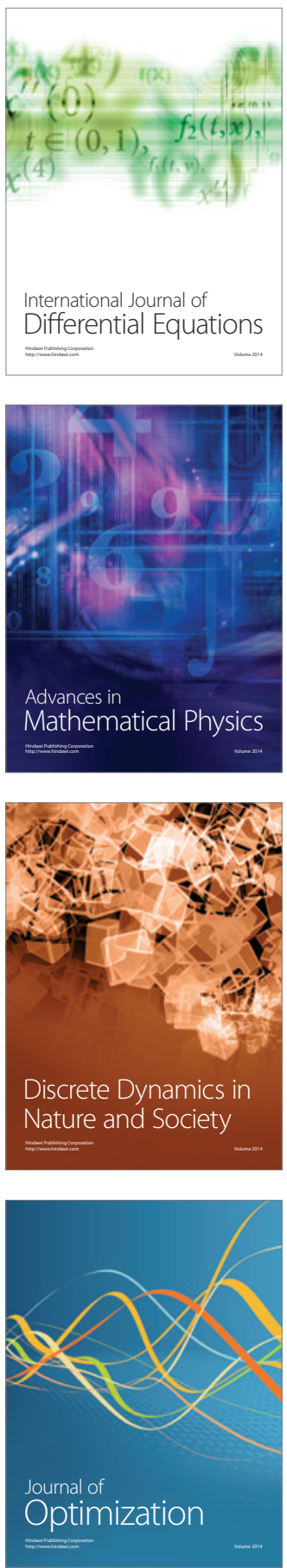